\title{
Critical sound attenuation of three-dimensional Ising systems
}

\author{
P.V.Prudnikov, V.V.Prudnikov \\ Omsk State University, Omsk, 644077 Russia
}

Received August 15, 2005, in final form November 14, 2005

\begin{abstract}
The field-theoretic description of dynamic critical effects of the disorder on acoustic anomalies near the temperature of second-order phase transition is considered for three-dimensional Ising-like systems. The calculations of sound attenuation in high temperature phase for pure and diluted Ising-like systems near the critical point are presented. The dynamic scaling function for critical attenuation coefficient is calculated. The effect of the quenched disorder on asymptotic behaviour of the critical ultrasonic anomalies is discussed.
\end{abstract}

Key words: renormalization group, disordered systems, field-theoretic approach, ultrasonic attenuation

PACS: $64.60 . \mathrm{Ak}, 64.60 . \mathrm{Fr}, 64.60 . \mathrm{Ht}, 43.35 . \mathrm{Cg}$

\section{Introduction}

The progress achieved in understanding the critical phenomena has largely been due to theoretical and experimental works devoted to studying the critical dynamics of condensed media. We have seen from experiments (figure 1) [1] that for a solid an anomaly peak of ultrasonic attenuation is observable in the vicinity of critical point. The critical anomalies exhibited by sound attenuation have long been recognized as an important study of dynamic critical phenomena.

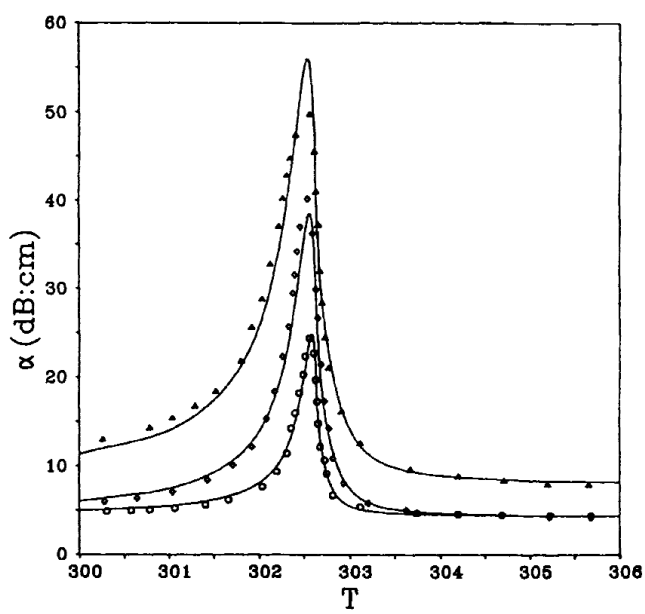

Figure 1. Ultrasound anomaly for experimental attenuation in $\mathrm{Rb}_{2} \mathrm{ZnCl}_{4}$ (dots) as function of temperature in comparison with the theoretical results (solid lines) [7].

The important physics processes are described by multi-spin correlation functions such as sound propagation and attenuation, fluctuations of energy density. Some experimental methods, such as EPR, NMR, propagation of ultrasonic waves are capable of studying the behaviour of multi-spin correlation functions. Ultrasonic methods permit simultaneous measurements of both static and 
dynamic properties. Measurements of sound velocities give information on the equilibrium properties, while measurements of sound attenuation yield the information on the dynamic properties of material.

The main difficulty in the theoretical discussion of critical propagation of sound waves consists in the estimation of the four-spin correlation function. The method, which is based on the representation of the four-spin correlation function through two-spin correlation functions by means of its decoupling, leads to overestimated values of critical fluctuations.

There are a lot of theories and phenomenological descriptions [1-8] of ultrasonic anomalies in solids with good agreement with the experiments [9-11] but real materials and crystals have many structural defects and the effect of such defects or disorder on the dynamic process of sound propagation in solid media should be taken into consideration.

Structural disorder, presence of impurities or other defects play an important role in real materials and physical systems. They may induce new types of phase transitions, universality classes as well as modify the transport properties of dynamic.

According to the Harris criterion [12], critical behaviour of Ising systems is changed by the presence of a weak quenched disorder. The problem of the effect of disorder on the critical sound propagation in Ising-like systems has been discussed in [13] with the use of $\varepsilon$-expansion in the lowest order of approximation. However, our pilot analysis of this phenomenon showed that in [13] there were not considered some diagrams which are indispensable for a correct description of disorder effect. Furthermore, our numerous investigations of pure and disordered systems performed in the two-loop and higher orders of approximation for a three-dimensional system directly and together with the use of series summation methods show that the predictions made in the lowest order of approximation, especially based on the $\varepsilon$-expansion can strongly differ from the real critical behaviour [14,15]. Therefore, the results from [13] must be reconsidered with the use of a more accurate field-theoretic approach in the higher orders of approximation.

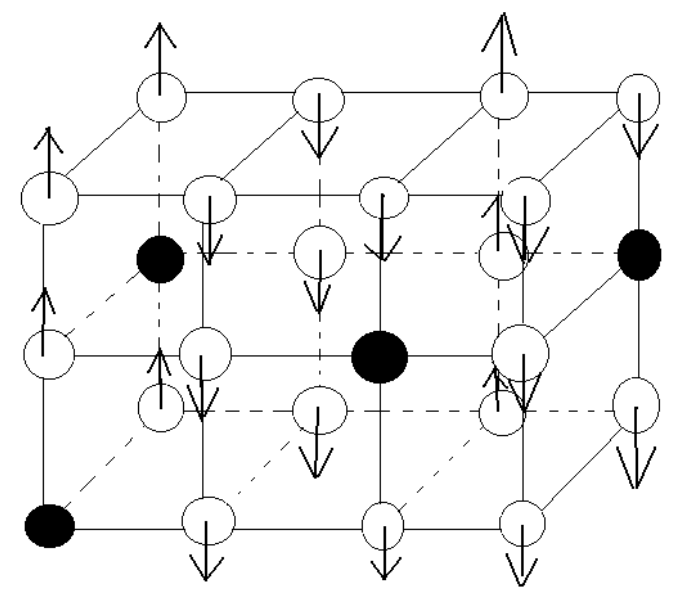

Figure 2. Three-dimensional disordered Ising system.

In this paper we have realized the correct field-theoretic description of dynamic effects of the disorder on acoustic anomalies near the temperature of second-order phase transition for a threedimensional Ising-like systems (figure 2) in the two-loop approximation.

\section{Model and RG analysis}

In this paper, we extended the model of phase transition in a disordered system with a coupling between nonfluctuating variables $[16,17]$ to the case (physically important for structural phase transitions) of a compressible three-dimensional Ising model with frozen-in lattice defects that is considered by the renormalization-group methods in the two-loop approximations. 
Interaction of the order parameter with elastic deformations plays a significant role in the critical behaviour of the compressible system. For the first time in [18] it was shown that the critical behaviour of the system with elastic degrees of freedom is unstable with respect to the connection of the order parameter with acoustic modes and the first-order phase transition is realized. However, the conclusions of [18] are only valid at low pressures. It was shown in [19] that in the range of high pressures, starting from a threshold value of pressure, the deformational effects induced by the external pressure lead to a change in the type of the phase transition.

The Hamiltonian of a disordered Ising model with allowance for elastic degrees of freedom may be specified as

$$
H=H_{\mathrm{el}}+H_{\mathrm{op}}+H_{\mathrm{int}}+H_{\mathrm{imp}}
$$

consisting of four contributions.

The elastic part is determined by

$$
H_{\mathrm{el}}=\frac{1}{2} \int \mathrm{d}^{\mathrm{d}} x\left[C_{11}^{0} \sum_{\alpha} u_{\alpha \alpha}^{2}+2 C_{12}^{0} \sum_{\alpha \beta} u_{\alpha \alpha} u_{\beta \beta}+4 C_{44}^{0} \sum_{\alpha<\beta} u_{\alpha \beta}^{2}\right]
$$

where $u_{\alpha \beta}(x)$ are components of the strain tensor and $C_{i j}^{k}$ are the elastic moduli.

$H_{\mathrm{op}}$ is a magnetic part in the appropriate Ginzburg-Landau form

$$
H_{\mathrm{op}}=\int \mathrm{d}^{\mathrm{d}} x\left[\frac{1}{2} \tau S^{2}+\frac{1}{2}(\nabla S)^{2}+\frac{1}{4} u_{0} S^{4}\right]
$$

where $S(x)$ is the Ising field variable which associated with the spin order parameter, $u_{0}$ is a positive constant and $\tau \sim\left(T-T_{\mathrm{c}}\right) / T_{\mathrm{c}}$ with the phase transition temperature $T_{\mathrm{c}}$.

The term $H_{\text {int }}$ describes the spin-elastic interaction

$$
H_{\text {int }}=\int \mathrm{d}^{\mathrm{d}} x\left[g_{0} \sum_{\alpha} u_{\alpha \alpha} S^{2}\right]
$$

which is bilinear in the spin order parameter and linear in deformations. The $g_{0}$ is the bare coupling constant.

The term $H_{\mathrm{imp}}$ of Hamiltonian determines the effect of disorder and it is considered as follows:

$$
H_{\mathrm{imp}}=\int \mathrm{d}^{\mathrm{d}} x\left[\Delta \tau(x) S^{2}\right]+\int \mathrm{d}^{\mathrm{d}} x\left[h(x) \sum_{\alpha} u_{\alpha \alpha}\right]
$$

where the random variables $\Delta \tau(x)$ and $h(x)$ are the local transition temperature fluctuations and induced random stress, respectively.

The Fourier transformed variables become

$$
u_{\alpha \beta}=u_{\alpha \beta}^{(0)}+V^{-1 / 2} \sum_{q \neq 0} u_{\alpha \beta}(q) \exp (\mathrm{i} q x)
$$

with $u(q)=\mathrm{i} / 2\left[q_{\alpha} u_{\beta}+q_{\beta} u_{\alpha}\right]$. We have to isolate in (6) tensor $u_{\alpha \beta}^{(0)}$ of homogeneous striction [18] and then make the integration in the partition function with respect to the nondiagonal components of the uniform part of the deformation tensor $u_{\alpha \beta}^{(0)}$ that are insignificant for the critical behaviour of the system in an elastically isotropic medium.

After all transformations [20] the effective Hamiltonian of the system becomes as follows: 


$$
\begin{aligned}
\tilde{H}= & \frac{1}{2} \int \mathrm{d}^{\mathrm{d}} q\left(\tau+q^{2}\right) S_{q} S_{-q}+\frac{1}{2} \int \mathrm{d}^{\mathrm{d}} q \Delta \tau_{-q} S_{q_{1}} S_{q-q_{1}} \\
& +\frac{1}{4} u \int \mathrm{d}^{\mathrm{d}} q S_{q_{1}} S_{q_{2}} S_{q_{3}} S_{-q_{1}-q_{2}-q_{3}}+\int \mathrm{d}^{\mathrm{d}} q q h_{-q} Q_{q} \\
& -\frac{1}{2} w \int \mathrm{d}^{\mathrm{d}} q\left(S_{q} S_{-q}\right)\left(S_{q} S_{-q}\right)-g \int \mathrm{d}^{\mathrm{d}} q q Q_{-q} S_{q_{1}} S_{q-q_{1}} \\
& +a \int \mathrm{d}^{\mathrm{d}} q q^{2} Q_{q} Q_{-q} .
\end{aligned}
$$

For a weak disorder, the distribution of random fields $\Delta \tau_{q}$ and $h_{-q}$ can be considered to be Gaussian

$$
P\left[\Delta \tau_{q}, h_{-q}\right]=A \int \mathrm{d}^{\mathrm{d}} q \exp \left[-\frac{1}{8 b_{1}} \Delta \tau_{q}^{2}-\frac{1}{8 b_{2}} h_{-q}^{2}-\frac{1}{8 b_{3}} \Delta \tau_{q} h_{-q}\right],
$$

where $A$ is the normalization constant and $b_{i}$ are positive constants which are proportional to the impurity concentration.

The critical dynamics of the system in the relaxation regime can be described by the Langevin equations [21] for the spin order parameter $S(q)$ and deformation variables $Q(q)$

$$
\dot{S}_{q}=-\Gamma_{0} \frac{\partial \tilde{H}}{\partial S_{-q}}+\xi_{q}+\Gamma_{0} h_{S}, \quad \ddot{Q}_{q}=-\frac{\partial \tilde{H}}{\partial Q_{-q}}-q^{2} D_{0} \dot{Q}_{q}+\eta_{q}+h_{Q},
$$

where $\Gamma_{0}$ and $D_{0}$ are the initial kinetic coefficients, $\xi_{q}(x, t)$ and $\eta_{q}(x, t)$ are Gaussian white noises.

The quantities of interest are the response functions $G(q, \omega)$ and $D(q, \omega)$ of spin and deformation variables, respectively. They can be obtained by linearization on corresponding fields

$$
\begin{aligned}
D(q, \omega) & =\delta\left[\left\langle Q_{q, \omega}\right\rangle\right] / \delta h_{Q}=\left[\left\langle Q_{q, \omega} Q_{-q,-\omega}\right\rangle\right], \\
G(k, \omega) & =\delta\left[\left\langle S_{q, \omega}\right\rangle\right] / \delta h_{S}=\left[\left\langle S_{q, \omega} S_{-q,-\omega}\right\rangle\right],
\end{aligned}
$$

where $\langle\cdots\rangle$ denotes the averaging over Gaussian white noises, $[\cdots]$ denotes the averaging over random fields $\Delta \tau_{q}$ and $h_{-q}$.

The response functions may be expressed in terms of self-energy parts

$$
G^{-1}(q, \omega)=G_{0}^{-1}(q, \omega)+\Pi(q, \omega), \quad D^{-1}(q, \omega)=D_{0}^{-1}(q, \omega)+\Sigma(q, \omega),
$$

where the free response functions $G_{0}(q, \omega)$ and $D_{0}(q, \omega)$ ar as follows:

$$
D_{0}(q, \omega)=1 /\left(\omega^{2}-a q^{2}-\mathrm{i} \omega D_{0} q^{2}\right), \quad G_{0}(q, \omega)=1 /\left(\mathrm{i} \omega / \Gamma_{0}+\left(\tau+q^{2}\right)\right) .
$$

Characteristics of critical sound propagation are defined by the response function $D(q, \omega)$. Thus, the coefficient of ultrasonic attenuation is determined through imaginary part of $\Sigma(q, \omega)$

$$
\alpha(\omega, \tau) \sim \omega \operatorname{Im} \Sigma(\omega) .
$$

In diagrammatic representation the expression for the self-energy part $\Sigma(q, \omega)$ in two-loop approximation is as follows:

$$
\Sigma(q, \omega)=4 g^{2}
$$


where we have used the following diagrammatic rules

$$
\begin{aligned}
G_{0}(q, \omega) & \\
\frac{2}{\Gamma_{0}} G_{0}(q, \omega) G_{0}(-q,-\omega) & \varkappa \\
v=\left[(\Delta \tau)^{2}\right] & \succ-\prec \prec .
\end{aligned}
$$

The Feynman diagrams involve momentum integrations on dimension $d$ (in our case $d=3$ ). Near the critical point the correlation length $\xi$ increases infinitely. When $\xi^{-1} \ll \Lambda$, where $\Lambda$ is a cutoff in momentum-space integrals (the cutoff $\Lambda$ serves to specify the basic length scale), the vertex functions are expected to display an asymptotic scaling behaviour for wave-numbers $q \ll \Lambda$. Therefore, one is lead to consider the vertex functions in the limit $\Lambda \rightarrow \infty$.

Now our purpose is that all variables become finite. We have applied the matching method introduced for statics in [23] and generalized for critical dynamics in [4]. At first, we use the dynamic scaling property of the response function

$$
D(q, \omega, \tau)=e^{2 l} D\left(q e^{l},\left(\omega / \Gamma_{0}\right) e^{z l}, \tau e^{(l / \nu)}\right),
$$

and then calculate the right-hand side of this equation for some value $l^{*}=l$, where not all the arguments vanish simultaneously [23]. The choice of $l^{*}$ is determined by

$$
\left(\omega_{l} / 2 \Gamma_{0}\right)^{4 / z}+\chi_{l}^{-2}=1 .
$$

The equation (14) guarantees that at least one of the arguments of the right-hand side of (15) is finite. The particular form of the matching condition (15) containing the exponents $z$ and $\nu$ permits an explicit solution for $l^{*}$

$$
e^{l}=\tau^{-\nu}\left[\left(1-b+b \tau^{-\alpha}\right)^{-2}+(y / 2)^{4 / z}\right]^{-1 / 4} \equiv \tau^{-\nu} F(y, \tau)
$$

In (16) b is an initial parameter and the abbreviation $y=\left(\omega / \Gamma_{0}\right) \tau^{-z \nu}$ is introduced and $F(y, \tau)$ is defined. The values for exponents $z, \alpha$ and $\nu$ were taken from [20] for a corresponding fixed point.

The response function $D\left(q e^{l},\left(\omega / \Gamma_{0}\right) e^{z l}, \tau e^{(l / \nu)}\right)$ on the right hand side of $(14)$ is represented by Dyson equation (12) and for self-energy part we obtain

$$
\frac{\operatorname{Im} \Sigma(\omega)}{\omega}=e^{l \frac{(\alpha+z \nu)}{\nu}} \frac{\operatorname{Im} \Sigma\left(\omega e^{z l}\right)}{\omega e^{z l}} .
$$

It may be argued [4] that condition (15) with a well-known expression for susceptibility [13] provides an infrared cutoff for all diverging values.

It was shown in the later theoretical works $[3,11]$ that in asymptotic regions the coefficient of attenuation is described by a simple scaling function of the variable $y$. The experimental investigations performed on three-dimensional crystals $\mathrm{Gd}$ [10] and $\mathrm{MnP}$ [11] confirmed the validity of the concepts of dynamic scaling.

\section{Scaling and asymptotic behaviour}

Thus after renormalization procedure (17) we can define the scaling relation in the form

$$
\operatorname{Im} \Sigma(\omega) / \omega=\tau^{-\alpha-z \nu} \phi(y),
$$

where $\phi(y)$ is a dynamic scaling function

$$
\begin{aligned}
\phi(y)= & \frac{g^{* 2} \Gamma_{0}}{\pi} \frac{F^{\alpha / \nu+1 / 2 \nu-z}}{y^{2}}\left\{1-\left[\frac{1}{2}\left(1+\frac{y^{2} F^{2 z-2 / \nu}}{4}\right)^{1 / 2}+\frac{1}{2}\right]^{1 / 2}\right\} \\
& -\frac{12 g^{* 2} u^{*} \Gamma_{0}^{2}}{\pi^{2}} \frac{F^{\alpha / \nu+1 / \nu-2 z}}{y^{3}}\left\{\left[\frac{1}{2}\left(1+\frac{y^{2} F^{2 z-2 / \nu}}{4}\right)^{1 / 2}-\frac{1}{2}\right]^{1 / 2}-\frac{y F^{z-1 / \nu}}{4}\right\} \\
& +\frac{8 g^{* 2} v^{*}}{(4 \pi)^{3}} \frac{F^{\alpha / \nu-z}}{y^{2}} \phi_{\mathrm{imp}}(y),
\end{aligned}
$$


where $v^{*}, g^{*}$, and $u^{*}$ are values of vertices in critical point [20], $\phi_{\mathrm{imp}}(y)$ is the numerically calculated contribution of diagrams in $\Sigma(\omega)$ with disorder inserting.

The dynamic scaling function $\phi(y)$ is plotted against $y$ on a double logarithmic scale for pure and disordered systems in figure 3 . We can determine two asymptotical regimes: "hydrodynamical" $\left(y \sim \omega \xi^{z} \sim(q \xi)^{z} \ll 1\right)$ with $\phi(y \rightarrow 0)=$ const and "critical" regime $\left(y \sim \omega \xi^{z} \gg 1\right)$ with characteristic power law $\phi_{\text {pure }}(y \rightarrow \infty) \sim y^{-0.96}, \phi_{\text {dis }}(y \rightarrow \infty) \sim y^{-0.78}$. Thus we have seen that the presence of disorder is irrelevant for the scaling behaviour in the hydrodynamic region with $y \ll 1$ but it produces a drastic effect in the critical region with $y \gg 1\left(T \rightarrow T_{\mathrm{c}}\right)$.

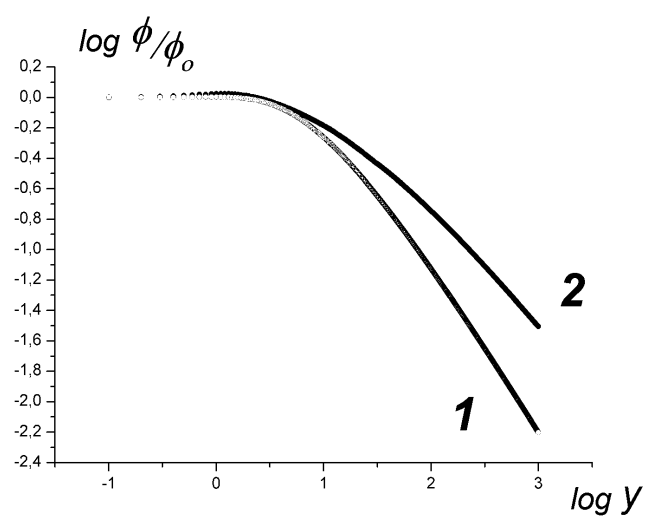

Figure 3. Scaling functions for the critical sound attenuation $\phi(y)$ in double-logarithmic plotting for pure (1) and disordered (2) Ising systems, $\phi_{0}=\phi(0)$.

We hope that these theoretical results will create a demand for the ultrasonic experimental investigations in the diluted Ising-like systems, for example $\mathrm{Fe}_{\mathrm{p}} \mathrm{Zn}_{1-\mathrm{p}} \mathrm{F}_{2}, \mathrm{Mn}_{\mathrm{p}} \mathrm{Zn}_{1-\mathrm{p}} \mathrm{F}_{2}$. In figure it was used that the presence of disorder causes the reduction of the phase transition temperature $T_{\mathrm{c}}$ in relation to the pure system. The model presentation of calculated attenuation coefficient for pure system is shown in figure 4 in comparison with experimental data (3) in $\mathrm{Gd}$ [8]. The adjustment on experimental data permited us to determine the value of theoretical parameter $\Gamma_{0}$ and calculate then the attenuation coefficient for disordered model. We must note that the observable differences with experimental results below $T_{\mathrm{c}}$ is explained by contribution of order parameter relaxation effects to attenuation which are presence always below $T_{\mathrm{c}}$. The relaxation effects are not considered in this paper. Whereas, the contribution of fluctuations to the attenuation coefficient is a relevant in the whole critical range.

Table 1. Asymptotic behaviour of the attenuation coefficient in the hydrodynamical and the critical regions.

\begin{tabular}{|l|l|l|}
\hline \multicolumn{1}{|c|}{ System } & \multicolumn{1}{c|}{ Region } & \multicolumn{1}{c|}{$\alpha(\omega, q)$} \\
\hline \hline Pure & hydrodyn. & $\omega^{2} \tau^{-1,38}$ \\
& critical & $\omega^{1.04} \tau^{-0.16}$ \\
\hline Disordered & hydrodyn. & $\omega^{2} \tau^{-1,44}$ \\
& critical & $\omega^{1.23} \tau^{-0,26}$ \\
\hline Pure $(\varepsilon$-exp. $)[5]$ & hydrodyn. & $\omega^{2} \tau^{-1,34}$ \\
& critical & $\omega^{0.858}$ \\
\hline
\end{tabular}

From equation (13) we find that the sound attenuation coefficient obeys the asymptotic scaling relation

$$
\alpha(\omega, \tau) \sim \omega^{2} \tau^{-\alpha-\nu z} \phi(y) .
$$




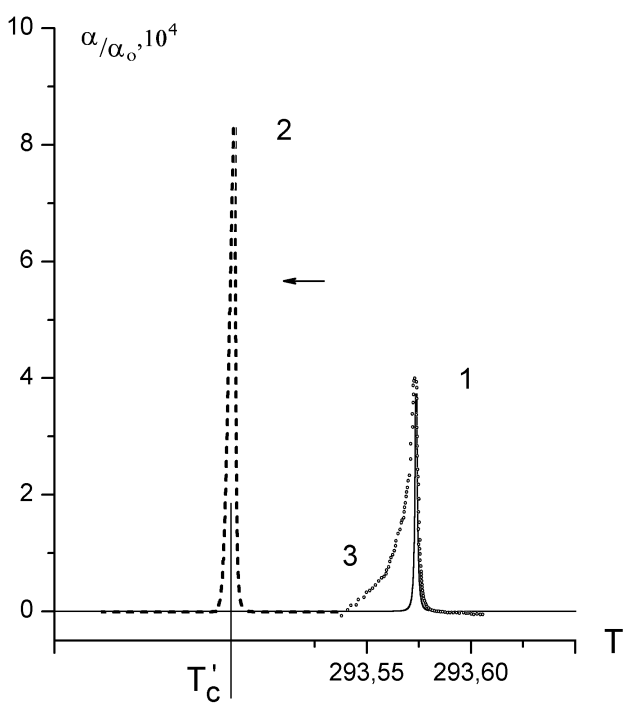

Figure 4. Thermal dependencies of the ultrasonic attenuation at the critical point for pure (1) and disordered (2) systems in comparison with experimental results (3) for Gd [8].

Based on the obtained dependencies (19) we defined the exponents of asymptotic behaviour of the attenuation coefficient in hydrodynamical and critical regions (table 1). The discrepancies between our results for the pure system and results in [5] are determinated by the use in [5] the critical exponents calculated on the basis of the $\varepsilon$-expansion, whereas we use the more accurate values of the critical exponents and vertices in fixed point for the three-dimesional system directly.

From the table we see that in the critical region the anomalies of the attenuation coefficient should be observe both in pure and disordered systems. However, for disordered systems the temperature and frequency dependent anomalies of the attenuation coefficient should be expressed stronger than in pure systems. These conclusions are also demonstrated in figure 4 .

The obtained theoretical estimates create a great demand for detecting the anomalies which are found to be induced by quenched disorder in ultrasonic experimental investigations of critical dynamics.

\section{Acknowledgement}

This work has been supported by the Russian Foundation for Basic Research through Grants (No. 04-02-17524, No. 04-02-39000 and No. 05-02-16188), and by Grant No. MK-8738.2006.2 of Russian Federation President.

\section{References}

1. Kamilov I.K., Aliev H.K., Physics-Uspehi, 1989, 41, 865 [Uspekhi Fizicheskikh Nauk, 1998, 168, 953].

2. Landau L.D., Khalatnikov I.M., Dokl.Akad. Nauk SSSR, 1954, 96, 496; Translated in "Collected Papers of Landau", ed. D. Ter Haar. Pergamon Press, New York, 1965.

3. Kawasaki K. Phase Transitions and Critical Phenomena, vol.5a, ed. C. Domb, M.S. Green. Academic press, London, 165, 1976; Kawasaki K. - In Proc. Conf. Internal Friction and Ultrasonic Attenuation in Solids. Tokyo, Tokyo University Press, 29, 1977.

4. Folk R., Iro H., Schwabl F., Zeitschrift fur Physik B, 1977, 27, 169; Dengler R., Iro H., Schwabl F., Phys. Lett A, 1985, 111, 121.

5. Schwabl F., Iro H., Ferroelectrics, 1981, 35, 215; Iro H., Schwabl F., Solid State Commun., 1983, 46, 205; Dengler R., Schwabl F., Zeitschrift fur Physik B, 1987, 69, 327. 
6. Pawlak A., J. Phys. Condens. Matter, 1989, 1, 7989; Pawlak A., Phys. Rev., 1991, B44, 5296.

7. Schorgg A.M., Schwabl F., Phys. Rev. B, 1993, 49, 11682.

8. Aliev H.K., Kamilov I.K., etc., Sov.Phys.JETP, 1989, 95, 1896 [Zh. Eksp. Teor. Fiz., 1989, 68, 1096].

9. Bhattacharjee J.K., Phys. Rev. B, 1982, 25, 3404; Ferrel R.A., Mirhashem B., Bhattacharjee J.K., Phys. Rev. B, 1987, 35, 4662.

10. Lüthi B., Moran T.J., Pollina R.G., J. Phys. Chem. Solids, 1970, 31, 1741; Moran T.J., Lüthi B., Phys. Rev. B, 1971, 4, 122.

11. Suzuki M., Komatsubara T., J. Phys. C, 1982, 15, 4559.

12. Harris A.B., J. Phys C., 1974, 7, 1671.

13. Pawlak A., Fechner B., Phys. Rev. B, 1989, 40, 9324.

14. Prudnikov V.V., Prudnikov P.V., Fedorenko A.A., Phys. Rev. B, 2000, 62, 8777; Phys. Rev. B, 2001, 63, 184201.

15. Pelissetto A., Vicari E., Phys. Rep., 2002, 368, 549; Folk R., Holovatch Yu., Yavors'kii T., Physics: Uspekhi, 2003, 46, 169.

16. Laptev V.M., Skryabin Yu.N., Phys. Status Solidi B, 1979, 91, K143.

17. Skryabin Yu.N., Shchanov A.V., Phys. Lett. A, 1997, 234, 147.

18. Larkin A.I., Pikin S.A., Sov. Phys. JETP, 1969, 29, 891 [Zh. Eksp. Teor. Fiz., 1969, 56, 1664].

19. Imry Y., Phys. Rev. Lett., 1974, 33, 1304.

20. Prudnikov V.V., Belim S.V., Physics of the Solid State, 2001, 43, 1353 [Fizika Tverdogo Tela, 2001, 43, 1299].

21. Meissner G., Ferroelectrics, 1980, 24, 27; Schwabl F., Iro H., Ferroelectrics, 1981, 35, 27.

22. Sh. Ma Modern Theory of Critical Phenomena. W.A. Benjamin Inc., London, 1976.

23. Nelson D.R., Phys. Rev. B, 1976, 14, 1123.

\title{
Затухання критичного звуку тривимірних ізингоподібних систем
}

\author{
П.В.Прудніков, В.В.Прудніков \\ Омський державний університет, м. Омськ 644 077, Росія \\ Отримано 15 серпня 2005 р., в остаточному вигляді - 14 листопада 2005 р.
}

Для тривимірних ізингоподібних систем здійснено теоретико-польовий опис динамічних і критичних ефектів безладу на акустичні аномалії поблизу температури фазового переходу другого роду. Представлено обчислення затухання звуку у високотемпературній фазі для чистих і розведених ізингоподібних систем поблизу критичної точки. Обговорюється вплив замороженого безладу на асимптотичну поведінку критичних ультразвукових аномалій.

Ключові слова: ренормалізаційна група, теоретико-польовий підхід, ультразвукове затухання

PACS: $64.60 . \mathrm{Ak}, 64.60 . \mathrm{Fr}, 64.60 . \mathrm{Ht}, 43.35 . \mathrm{Cg}$ 